\title{
Ciencia política en Costa Rica: BÚSQUEDA DE IDENTIDAD DISCIPLIN ARIA*
}

\author{
Ronald Alfaro Redondo \\ Universidad de Costa Rica, Costa Rica \\ J orge Vargas Cullell \\ Universidad de Notre Dame, Estados Unidos
}

\begin{abstract}
Resumen
Este artículo explora las principales características de la ciencia política en Costa Rica a lo largo de poco más de 35 años de existencia. Para ello aporta una periodización del desarrollo histórico de la disciplina, basada en la clasificación de los temas y estilos de investigación utilizados en la producción académica. El trabajo aporta evidencias empíricas que dan cuenta de cómo la politología en el país se encuentra actualmente enfrascada en la búsqueda de identidad disciplinaria en el plano teórico y fundamentalmente en el plano metodológico.
\end{abstract}

Abstract

This article explores the main characteristics of political science in Costa Rica over the last 35 years. It proposes a periodization of the historical development of the discipline, based on atopical classification and the research styles used in academic production. This paper uses empirical evidence to show how the discipline is currently, confronted with involved in the search for disciplinary identity at both a theoretical and methodological level.

PALABRAS CLAVE • Ciencia Política • Costa Rica • Identidad Disciplinaria • Desarrollo Histórico • Producción Académica

\section{INTRODUCCIÓN}

Si como disciplina académica la ciencia política en Costa Rica es de un desarrollo relativamente reciente, como práctica distintiva de investigación lo es todavía más. La primera -y única hasta la fecha- escuela universitaria fue fundada en la Universidad de Costa Rica en 1968 bajo el nombre de Escuela de Ciencias Políticas, en una época similar a la creación de otras escuelas de ciencias sociales como la de sociología y la de psicología. Posteriormente, en 1977 fue creada la Escuela de Relaciones Internacionales de la Universidad Nacional Autónoma. Recientemente, se han fundado otras escuelas de Relaciones Internacionales en universidades privadas, pero son todavía incipientes. Como apunte histórico, cabe anotar que en el siglo XIX existió la Facultad de Leyes y Ciencias Políticas en la Universidad de Santo Tomás. Sin embargo, esta universidad fue cerrada en 1888 y no fue sino hasta 1940, con la apertura de la Universidad de Costa Rica, que el país volvió a tener una universidad. En el interregno funcionaron las escuelas de Derecho, Farmacia y Agronomía como entidades independientes.

* Los autores agradecen la colaboración de Iván Acuña Chaverri, quien recopiló la información sobre los libros, revistas y tesis de grado sobre los que se basa el presente artículo. 
Una revisión de 235 libros y revistas publicados en Costa Rica y de 151 tesis de grado ${ }^{1}$ permite concluir que la mayoría de la producción intelectual ocurre en los últimos 15 años. Los estudios en ciencias políticas se caracterizan por recurrir a métodos tradicionales de investigación como la interpretación documental (reprocesamiento de fuentes documentales como libros y periódicos). Con excepción de los estudios hechos sobre Costa Rica por politólogos extranjeros², el empleo sistemático de técnicas de observación cuantitativas y cualitativas no sólo es reciente, sino escaso. Buena parte de la producción, además, tiene un carácter ensayístico o bien descriptivo. En este breve artículo nos enfocamos en aportar la evidencia que sustenta estas afirmaciones ${ }^{3}$.

\section{LIBROS Y ARTÍCULOS DE REVISTAS PUBLICADOS EN COSTA RICA}

Un examen de la producción de libros y artículos en revistas sobre temas de ciencia política en Costa Rica permite distinguir dos fases. La primera comprende entre 1970-1989 y se caracteriza por muy pocas publicaciones: 38 en total, en promedio menos de dos obras por año (Tabla 1$)^{4}$. Esta escasa producción se concentra en dos temas: los estudios sobre el sistema electoral y partidos políticos (14 obras) y sobre teoría política e historia de las ideas (9 obras). En estos años, una parte importante de los estudios sobre la política costarricense se hace desde fuera de las ciencias políticas, desde disciplinas como la historia y la sociología, o bien desde centros de investigación con profesionales formados en otros campos.

1 Esta recopilación incluyó una revisión de los siguientes centros documentales: Biblioteca Carlos Monge Alfaro y Biblioteca Eugenio Fonseca Tortós de la Universidad de Costa Rica y Biblioteca J oaquín García Monge de la Universidad Nacional. Cabe advertir enfáticamente que no se trata de una recopilación bibliográfica exhaustiva. Esta limitación es naturalmente, responsabilidad nuestra. En ello influyeron, además, las dificultades para delimitar las búsquedas en relación con textos de otras disciplinas como la sociología y la historia, así como los recursos disponibles para este proyecto. Estamos concientes que la producción de varios autores no está adecuadamente representada -en algunos casos de manera importante- y esperamos en el futuro subsanar estas limitaciones. Empero, cabe indicar que se trata de la más amplia recopilación biblográfica sobre estudios políticos que hasta la fecha existe en el país. Por ello, las conclusiones a las que arribamos no son definitivas y están sujetas a cambios conforme se logre perfeccionar la base de datos. La intensidad de las tendencias que identificamos puede variar (aunque no creemos que se modifique su sentido).

2 Entre estos cabe destacar los estudios que desde la década de 1970, Mitchell Seligson y J ohn Booth han efectuado sobre Costa Rica, solos o con otros colegas, en especial el estadístico costarricense Miguel Gómez. Seligson escribe sobre la cultura política (Seligson, 1980, 1999, 2002; Seligson y Booth, 1993; Seligson y Gómez, 1987; Seligson y Muller, 1990), Booth sobre ésta y, en general sobre el desarrollo democrático del país (Booth, 1995, 1998; Booth y Seligson, 1994, 2005). A estos cabe agregar las investigaciones de Fabrice Lehoucq sobre la política costarricense en la primera mitad del siglo XX, solo o con el historiador costarricense Iván Molina (Lehouq, 1992, 1995, 1998; Molina y Lehouq, 1999) y las de Bruce Wilson sobre la evolución reciente del país y, en sus últimas obras, su énfasis sobre la administración de justicia (Wilson, 1994, 1998, 2004; Wilson y Rodríguez Cordero, 2004). Otros trabajos relevantes son los de Peeler y colegas acerca de elites y democratización (Peeler, 1985, 1991), Clark sobre el desarrollo democrático del país (Clark, 2001) y los de Deborah Yashar y Richard Mahoney sobre historia política (Mahoney, 2001; Yashar, 1997).

3 Debido a las limitaciones de la base de datos indicada en la nota 2 y al muy estrecho espacio disponible nos concentramos en la identificación de tendencias generales en la producción intelectual en la disciplina de ciencias políticas en Costa Rica, con obvio énfasis en autores costarricenses, aunque no exclusivo. En general, procuramos incluir toda obra publicada dentro del país, sea por un autor nacional o extranjero. Deliberadamente hemos evitado la tentación de efectuar una síntesis sobre la historia de los intelectuales especializados en ciencias políticas, o cualquier intento por efectuar un balance sobre el "estado del arte" en las ciencias políticas costarricenses, sus hitos, áreas de debate, alcances y limitaciones.

$4 \quad$ Las primeras obras en la disciplina de ciencias políticas se caracterizan por dos enfoques muy distintos. Por un lado, las obras de intepretación general del desarrollo político influidas por la teoría marxista de las clases sociales (Camacho, 1977; Cerdas, 1972, 1974), los imperativos de la política pública (Arias Sánchez, 1977; Daremblum y Stone, 1975), u obras descriptivas sobre acontecimientos u organizaciones políticas (Araya, 1982). Excepciones tempranas de esta situación son las obras de Arias Sánchez sobre los grupos de presión en Costa Rica, donde hay una aplicación de una teoría pluralista de la política (Arias Sánchez, 1971) y de Mario Carvajal, sobre las actitudes de dirigentes políticos (Carvajal, 1978). 
En el primer caso, cabe destacar los trabajos de los historiadores Marielos Aguilar y Jorge Mario Salazar, de los sociólogos J osé Luis Vega Carballo, Manuel Rojas y Ana Sojo. Salazar escribe sobre la historia de los partidos y el sistema de partidos políticos (Salazar, 1981, 1990); Aguilar sobre los derechos civiles (Aguilar, 1989a, 1989b); Vega Carballo aplica la teoría de la dependencia a Costa Rica y escribe sobre partidos políticos (Vega Carballo, 1978, 1982, 1986); Manuel Rojas sobre el desarrollo democrático (Rojas, 1990, 1989) y Ana Sojo y J orge Rovira efectúan estudios de economía política sobre la intervención del Estado en la economía (Rovira, 1988; Sojo, 1984).

En lo que a producción de centros de investigación se refiere durante este primer periodo, vale la pena reseñar las activas producciones del Centro de Estudios para la Acción Social (CEPAS), del Instituto Centroamericano de Ciencias Sociales (ICES) y del Centro Alforja durante la década de 1980. Hasta su cierre a inicios de la década de 1990, CEPAS produjo informes de coyuntura y estudios especializados en política, sociología y economía. Por su parte el ICES produjo varias obras sobre la situación política del país, especialmente en relación con los conflictos centroamericanos, y auspició estudios sobre la evolución de los derechos civiles en Costa Rica. Alforja se caracterizó por la producción de textos para la educación popular, muchos de los cuales eran interpretaciones sobre las instituciones y los actores políticos. En contraste con estos centros, ya desaparecidos, desde 1975 funciona el Centro de Investigación y Adiestramiento Político y Administrativo (CIAPA), la mayoría de cuyos socios principales son cientistas políticos o con amplia experiencia en la disciplina (Samuel Stone, Constantino Urcuyo, Rodolfo Cerdas, Jaime Daremblum). Aunque se trata de destacados politólogos, con amplias e influyentes producciones editoriales, la mayoría de las obras por cuenta de CIAPA se publican a partir de la década de 1990 (http:// www.ciapa.org).

La segunda fase de producción intelectual abarca los años 1990-2005 y, a diferencia de la anterior, se destaca por un fuerte incremento en la cantidad de publicaciones libros y revistas. En total se logró recopilar 197 libros y revistas, un promedio de 13,3 obras por año -siete veces superior al de la fase anterior-. Con mayor o menor intensidad, en todos los temas ocurrió una ampliación editorial. Lo más interesante, sin embargo, son los cambios de énfasis. En ambos períodos, las publicaciones sobre el sistema electoral y de partidos políticos siguieron siendo las más frecuentes. Mas en la segunda fase este énfasis fue aún mayor: más del $50 \%$ de las obras de este período se dedican a este tema (101 de las 197). En estos años las Relaciones Internacionales surgen como una temática relevante (25 obras). En cambio, otros temas como los de historia política, los estudios jurídicos y el de teoría política e historia de las ideas son relativamente relegados, pues sus proporciones dentro de la producción editorial descienden significativamente. 
TABLA 1: Tema de las publicaciones (libros y revistas) de ciencia política en Costa Rica según periodo $1970-2005^{\text {al }}$

\begin{tabular}{|c|c|c|c|c|c|c|c|c|}
\hline \multirow[t]{2}{*}{ Tema $^{b /}$} & \multicolumn{8}{|c|}{ Período } \\
\hline & $\begin{array}{c}1970- \\
1974\end{array}$ & $\begin{array}{c}1975- \\
1979\end{array}$ & $\begin{array}{c}1980- \\
1984\end{array}$ & $\begin{array}{c}1985- \\
1989\end{array}$ & $\begin{array}{c}1990- \\
1994\end{array}$ & $\begin{array}{c}1995- \\
1999\end{array}$ & $\begin{array}{c}2000- \\
2005\end{array}$ & $\begin{array}{c}\text { Total } \\
\text { general }\end{array}$ \\
\hline \multirow[t]{2}{*}{ Estudios J urídicos } & & 1 & & & 1 & 6 & & 8 \\
\hline & & (12) & & & (12) & $(75)$ & & $(100)$ \\
\hline \multirow[t]{2}{*}{ Historia Política } & & & 1 & 4 & 5 & 2 & 2 & 14 \\
\hline & & & (7) & (29) & (36) & (14) & (14) & $(100)$ \\
\hline Políticas Públicas y & & 4 & 1 & 2 & 4 & 12 & 10 & 33 \\
\hline Política Económica & & (12) & (3) & (6) & (12) & (36) & (30) & $(100)$ \\
\hline Relaciones & 1 & & 1 & & 11 & 9 & 5 & 27 \\
\hline internacionales & (4) & & (4) & & $(41)$ & (33) & (18) & $(100)$ \\
\hline Sistema electoral y & 2 & 5 & & 7 & 21 & 39 & 41 & 115 \\
\hline partidos políticos & (2) & (4) & & (6) & (18) & (34) & (36) & $(100)$ \\
\hline Teoría Política e & & 1 & 2 & 6 & 5 & 14 & 7 & 35 \\
\hline historia de las ideas & & (3) & (6) & (17) & (14) & $(40)$ & (20) & $(100)$ \\
\hline \multirow[t]{2}{*}{ Otro } & & & & & & 1 & 2 & 3 \\
\hline & & & & & & (33) & (67) & 100,0 \\
\hline \multirow[t]{2}{*}{ Total general } & 3 & 11 & 5 & 19 & 47 & 83 & 67 & 235 \\
\hline & (1) & (5) & (2) & (8) & (20) & (35) & (28) & $(100)$ \\
\hline
\end{tabular}

Porcentajes: entre paréntesis ( ). Por efectos de redondeo la suma de porcentajes no da 100.

Fuente: elaboración propia, 2005 a partir de la revisión hecha por Ronald Alfaro e Iván Acuña.

a) Del 1/1/1970 al 30/4/2005

b/ Análisis jurídico: corresponde a casos en los que se enfatiza en el análisis del marco legal, procedimientos y disposiciones legales, teoría del derecho, administración de la justicia, así como la vinculación de éstos con un análisis de índole político. Historia política: el énfasis de la publicación es la combinación de categorías teóricas pertenecientes a la historia y la ciencia política. Políticas públicas y política económica: contempla casos en los que se abordan aspectos como diseño, formulación y evaluación de políticas públicas, así como estudios con una perspectiva que vincula teóricamente economía y política. Relaciones internacionales: se trata de publicaciones con un enfoque teórico basado en temas como política internacional, política exterior, relaciones internacionales, diplomacia, entre otros. Sistema electoral y partidos políticos: se agrupan publicaciones cuyo abordaje teórico pone énfasis en categorías conceptuales como sistema político, partidos políticos, comportamiento electoral y sistema electoral. Teoría política e historia de las ideas: corresponde a publicaciones cuya perspectiva teórica da importancia a la comparación de categorías conceptuales entre autores, realidades y contextos, así como la evolución conceptual en el tiempo. Otras: se incluyen publicaciones cuya especificidad no permite ubicarlas en alguna de las categorías anteriores.

Si se analizan en conjunto las obras, sin distinguir las fases antes mencionadas, puede apreciarse que el estilo de investigación más utilizado en los estudios politológicos es el reprocesamiento de fuentes documentales (análisis sistemáticos de documentos, periódicos y discursos, entre otros). Este estilo de investigación ha sido empleado en 76 de los 235 libros y revistas recopiladas. Le 
sigue en orden de frecuencia el uso de fuentes secundarias (casos en los que se citan datos existentes sin agregar nueva información o transformarla), que fue empleado en 73 ocasiones. A esto debemos sumar lo que hemos denominado las investigaciones sobre problemas teóricos, es decir, cuando un autor realiza una interpretación crítica del pensamiento de uno o varios intelectuales (38 obras). Los métodos cuantitativos de investigación son, por lo tanto, un estilo bastante minoritario. La aplicación e interpretación de encuestas y el reprocesamientos de registros administrativos para crear indicadores han sido empleadas en menos de 30 obras de las 235 recopiladas. En resumen, casi el $80 \%$ (197 de 235) de los estudios en ciencias políticas se limitan a las técnicas documentales -análisis textual o extracción de información.

Las diferencias en los estilos de investigación aplicados por los estudios politológicos en las dos fases que hemos distinguido son importantes, pero no hay evidencia de una ruptura metodológi$\mathrm{ca}^{5}$. En términos generales, las técnicas documentales predominaron en ambos períodos: entre 1970 y 1989, 34 de las 38 obras analizadas (89\%) reprocesaron fuentes documentales, utilizaron fuentes secundarias o efectuaron investigación teórica; esta proporción descendió ligeramente entre 1990-2005, cuando 153 de las 197 obras lo hicieron (78\%). Sin embargo, estos números encubren una importante diferencia. Mientras que en la primera fase el estilo predominante fue el uso de fuentes secundarias, es decir, la extracción y trascripción de datos elaborados por otros (20 de las 38 obras, 53\%), este estilo pierde fuerza en la segunda fase, cuando poco más de una cuarta parte de las obras se limitaron a ello (53 de 197, 27\%). Dentro de la familia de análisis documental, en cambio, el estilo predominante es el análisis sistemático de textos, que encontramos en 65 de las 197 obras de este período (35\%). Ello ciertamente denota un avance, pues supone una labor más activa por parte de los autores en materia de reprocesamiento de fuentes ${ }^{6}$.

Al igual que en la fase anterior, entre 1990 y 2005, la aplicación e interpretación de encuestas y el reprocesamiento de registros administrativos para crear indicadores son estilos minoritarios. Sin embargo, es importante anotar que la proporción de obras que lo utilizaron más que se duplica, aunque a partir de una base muy exigua ${ }^{7}$. En resumen, durante la segunda fase (1990-2005) es posible identificar una mayor pluralidad de estilos de investigación empleados. No sólo se trata de más estilos, sino que aquellos que ya venían siendo aplicados tímidamente, empezaron a ser empleados con más frecuencia.

5 Utilizamos la noción de ruptura metodológica para denotar una transformación radical en la familia de métodos predominantes para la investigación de los sistemas, procesos, instituciones, actores y eventos políticos. Para efectos de este artículo reconocemos tres familias: métodos cuantitativos (uso de encuestas, reprocesamiento de registros administrativos para la elaboración de indicadores); métodos cualitativos (entrevistas, grupos focales, juicios grupales ponderados); análisis documental (extracción de información e interpretación de textos). Una ruptura ocurre cuando existe una alteración substancial en las preferencias (en el sentido de escogencia) entre familias de métodos para la observación, registro e interpretación de la información.

6 Una línea distinta de trabajo es la que Constantino Urcuyo ha desarrollado en los últimos diez años. Sus ensayos están orientados a formular propuestas de reestructuración institucional del sistema político costarricense (Urcuyo, 2003).

7 Dentro de una orientación más cuantitativa destacan los trabajos realizados por centro de investigaciones PROCESOS en materia de cultura política (Rodriguez y Castro, 2002; Rodríguez, Castro, y Espinoza, 1998; Rodríguez, Castro, y Madrigal, 2004); el Instituto de Investigaciones Sociales de la Universidad de Costa Rica en materia electoral (Fournier, M., 2002; Fournier, M. V., Gutiérrez y Cruz, 2002; Raventós Vorst et al., 2004), y el esfuerzo del Programa Estado de la Nación por construir indicadores políticos (Programa Estado de la Nación, 2001a, 2003b, 2004). También es destacable la incidencia de centros de educación extranjeros en la ejecución de investigaciones políticas. Dentro de ellas debe mencionarse los estudios de la Universidad de Salamanca sobre partidos y élites políticas, dirigido por Manuel Alcántara y el Proyecto de Opinión Pública de América Latina que dirige Mitchell Seligson. 
TABLA 2: Estilos de investigación de las publicaciones (libros y revistas) de ciencia política en Costa Rica según periodo 1970-2005 al

\begin{tabular}{|c|c|c|c|c|c|c|c|c|}
\hline \multicolumn{4}{|c|}{ Estilo de Investigación b/ } & \multicolumn{4}{|c|}{ Período } & \multirow[b]{2}{*}{$\begin{array}{l}\text { Total } \\
\text { general }\end{array}$} \\
\hline & $\begin{array}{l}1970- \\
1974\end{array}$ & $\begin{array}{l}1975- \\
1979\end{array}$ & $\begin{array}{c}1980- \\
1984\end{array}$ & $\begin{array}{c}1985- \\
1989\end{array}$ & $\begin{array}{c}1990- \\
1994\end{array}$ & $\begin{array}{c}1995- \\
1999\end{array}$ & $\begin{array}{l}2000- \\
2005\end{array}$ & \\
\hline Aplicación e & & 2 & & & 1 & 6 & 6 & 15 \\
\hline \multicolumn{8}{|l|}{ de encuestas } & $(100)$ \\
\hline Investigación & & & & & & 1 & 8 & 9 \\
\hline empírica & & & & & & $(11)$ & (89) & $(100)$ \\
\hline Investigación sobre & & 1 & 1 & 5 & 7 & 13 & 11 & 38 \\
\hline problema teórico & & (3) & (3) & (13) & 18 & (34) & (28) & $(100)$ \\
\hline \multirow{2}{*}{\multicolumn{2}{|c|}{$\begin{array}{l}\text { Reprocesamiento de } \\
\text { fuentes documentales }\end{array}$}} & 2 & 2 & 3 & 13 & 35 & 21 & 76 \\
\hline & & (3) & (3) & (4) & 17 & $(46)$ & $(27)$ & $(100)$ \\
\hline Uso de fuentes & 2 & 6 & 2 & 10 & 17 & 22 & 14 & 73 \\
\hline secundarias & (3) & (8) & (3) & (14) & 23 & (30) & (19) & $(100)$ \\
\hline \multirow[t]{2}{*}{ Otros } & 1 & & & 1 & 9 & 6 & 7 & 24 \\
\hline & (4) & & & (4) & 38 & $(25)$ & (29) & $(100)$ \\
\hline \multirow[t]{2}{*}{ Total general } & 3 & 11 & 5 & 19 & 47 & 83 & 67 & 235 \\
\hline & (1) & (5) & (2) & (8) & (20) & (35) & (28) & $(100)$ \\
\hline
\end{tabular}

Porcentajes: entre paréntesis (). Por efectos de redondeo la suma de porcentajes no da 100.

FUENTE: elaboración propia, 2005 a partir de la revisión hecha por Ronald Alfaro e Iván Acuña.

a/ Del 1/1/1970 al 30/4/2005

b/ Categorías: 1. Aplicación e interpretación de encuestas: incluye aquellas publicaciones en las cuales se aplicó encuestas para la investigación o se reprocesó la información de una encuesta. No contempla casos en los que únicamente se citan datos de encuestas. 2. Investigación empírica: comprende publicaciones en las cuales se reprocesaron registros administrativos con la finalidad de crear nuevos indicadores. 3. Investigación sobre problema teórico: se trata de casos basados en el análisis y discusión de textos mediante la modalidad de un debate con autores y conceptos. 4. Reprocesamiento de fuentes documentales: corresponde a análisis sistemáticos a lo largo de un período específico de documentos, periódicos y discursos, entre otros. 5 . Uso de fuentes secundarias: se trata de casos en los que se citan datos existentes sin agregar nueva información o transformarla. 6. Otros: se incluyen publicaciones cuya especificidad no permite ubicarlas en alguna de las categorías anteriores.

La información aportada permite un último comentario. Si existe un tema de alto desarrollo y sofisticación metodológica en las ciencias políticas contemporáneas es el estudio de sistemas electorales y partidos políticos. La aplicación de la teoría de juegos, la adaptación de técnicas econométricas y la aplicación de análisis multivariados prevalecen hoy en día. Pareciera ser que la disciplina en Costa Rica comparte el énfasis temático en materia electoral y partidos característico de esta disciplina, pero su arsenal metodológico sigue siendo, en términos generales, rudimentario. 


\section{TRABAJ OS FINALES DE GRADUACIÓN ${ }^{8}$}

Los trabajos finales de graduación para optar al título de licenciatura en la Escuela de Ciencias Políticas de la Universidad de Costa Rica, el principal centro de estudios de esta disciplina en el país, constituyen una valiosa e importante fuente de investigaciones políticas. En total, se registraron 151 trabajos finales de graduación de grado (al 30 de abril 2005).

En materia de estilos de investigación, estos trabajos muestran un comportamiento similar al señalado para los libros y artículos de revista. Las técnicas documentales de análisis sistemático de textos o la extracción simple de información dominan el panorama a lo largo del período 19702005 (84\% de los 151 casos). Quizá, como era de esperarse, las limitaciones metodológicas son aun más acentuadas: 92 trabajos (61\%) se limitan a transcribir datos secundarios como apoyo a sus elaboraciones, principalmente en temas como políticas públicas y política económica, así como relaciones internacionales (Tabla 3).

En términos temáticos, sin embargo, las diferencias son importantes, pese a que la materia electoral y de partidos políticos tiene una importante presencia, los trabajos finales de graduación en políticas públicas y política económica han venido ganando terreno y representan poco más de una tercera parte del total de éstos. Por una parte, muchos de los trabajos son estudios "de caso": una organización social, una política pública, una institución, un partido o un evento -un gane o pérdida electoral-. Por otra parte, hay una importante atención a los estudios sobre medios de comunicación colectiva y grupos sociales vulnerables que no está tan presentes en los libros y artículos de revista compilados.

En general, tanto los estilos de investigación como los temas prevalentes en los trabajos de graduación parecieran responder a una respuesta (lógica) a la necesidad de desarrollar investigaciones rápidas y de bajo costo. graduación en cuatro modalidades: tesis (la más conocida y la más utilizada), seminario de graduación, proyecto de graduación y práctica dirigida. Estas modalidades se diferencian entre sí principalmente en aspectos de abordaje teórico y metodológico. La mayor parte de los trabajos finales de graduación de la Escuela de Ciencias Políticas se han desarrollado bajo la modalidad tesis, sin embargo, también se registraron los casos existentes de otras modalidades que, como se apuntó anteriormente, se trata de pocos casos. 
TABLA 3: Tema y estilos de investigación de los trabajos finales de graduación de ciencia política en Costa Rica 1970-2005 al

\begin{tabular}{|c|c|c|c|c|c|c|c|}
\hline \multirow[t]{2}{*}{ Tema b/ } & \multicolumn{7}{|c|}{ Estilos de investigación cl } \\
\hline & 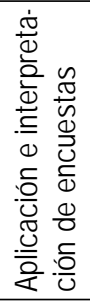 & 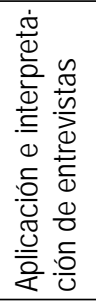 & 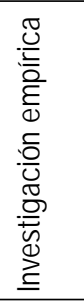 & 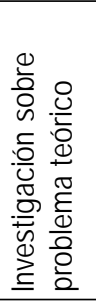 & 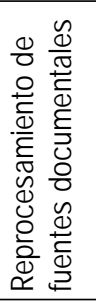 & 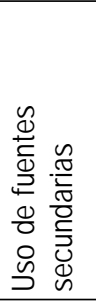 & $\begin{array}{l}\overline{0} \\
\bar{d} \\
\overline{0} \\
\frac{0}{0} \\
\overline{0} \\
0\end{array}$ \\
\hline \multirow[t]{2}{*}{ Grupos sociales vulnerables } & 2 & 4 & & & 2 & 1 & 9 \\
\hline & (22) & $(44)$ & & & $(22)$ & (11) & $(100)$ \\
\hline \multirow{2}{*}{$\begin{array}{l}\text { Organizaciones sociales y } \\
\text { medios de comunicación }\end{array}$} & 1 & & & & 3 & 8 & 12 \\
\hline & (8) & & & & $(25)$ & $(67)$ & $(100)$ \\
\hline Políticas Públicas y Política & 3 & 1 & & & 10 & 42 & 56 \\
\hline Económica & (5) & $(2)$ & & & (18) & (75) & $(100)$ \\
\hline \multirow[t]{2}{*}{ Relaciones internacionales } & & 1 & 1 & & 2 & 23 & 27 \\
\hline & & (4) & $(4)$ & & (7) & (85) & $(100)$ \\
\hline \multirow{2}{*}{$\begin{array}{l}\text { Sistema electoral y } \\
\text { partidos políticos }\end{array}$} & 3 & & 3 & & 14 & 13 & 33 \\
\hline & (9) & & (9) & & $(42)$ & (39) & $(100)$ \\
\hline \multirow{2}{*}{$\begin{array}{l}\text { Teoría Política e historia } \\
\text { de las ideas }\end{array}$} & & & & 3 & 3 & 1 & 7 \\
\hline & & & & (43) & $(43)$ & $(14)$ & $(100)$ \\
\hline \multirow[t]{2}{*}{ Otro } & 1 & & 2 & & & 4 & 7 \\
\hline & (14) & & $(29)$ & & & $(57)$ & 100 \\
\hline \multirow[t]{2}{*}{ Total general } & 10 & 6 & 6 & 3 & 34 & 92 & 151 \\
\hline & (7) & (4) & (4) & (2) & (23) & $(61)$ & $(100)$ \\
\hline
\end{tabular}

Porcentajes: entre paréntesis ( ). Por efectos de redondeo la suma de porcentajes no da 100.

Fuente: elaboración propia, 2005 a partir de la revisión hecha por Ronald Alfaro e Iván Acuña.

a) Del 1/1/1970 al 30/4/2005

b/ Para el detalle de las categorías véase Tabla 1

c) Para el detalle completo de las categorías véase Tabla 2.

\section{MERCADO LABORAL: UN ASPECTO POCO EXPLORADO}

En Costa Rica no se cuenta con estudios especializados que aborden de manera sistemática9 las principales características del mercado laboral de la ciencia política, lo cual constituye un gran

9 El último estudio fue realizado a solicitud de las autoridades universitarias en el año 1986 por la OPES, el cual se titula Estudio exploratorio del mercado de trabajo en el campo de las ciencias políticas. Este estudio determinó que un $46,2 \%$ de los egresados no trabajaban en puestos relacionados con su profesión. Los politólogos consultados se desempeñaban en tareas muy diversas tales como consultor, asesor, investigador, analista y docente. 
vacío. No obstante, algunos resultados del estudio "La situación laboral y otras características de los graduados de las universidades estatales"10, cuya más reciente publicación incluye los graduados del 2001, son de interés para el presente artículo.

En el año 2001 se graduaron un total de 38 politólogos (26 en Bachillerato, 3 en Licenciatura y 9 en Maestría). En la muestra de la Oficina de Planificación de la Educación Superior (OPES) tomaron parte los 26 graduados del Bachillerato de Ciencias Políticas, que son una pequeña parte de los 4.253 graduados considerados en la muestra. 21 de los 26 encuestados (un $80 \%$ ) manifestaron encontrarse trabajando en el momento del estudio, de los cuales 12 opinaron que trabajaban en labores relacionadas con la disciplina, para quienes la relación del trabajo con su campo profesional es de categoría media (en una escala de 1 a 5, con un valor de 3,0). Según el estudio, la mediana del salario mensual que reciben los bachilleres en ciencia política que trabajan es de US\$500 (al tipo de cambio de 473,5 colones por dólar). En cuanto al desarrollo organizacional actual de la disciplina, podría hablarse de una comunidad de cientistas políticos incipiente. Como muestra de ello, hasta hace pocos años, una buena parte de los profesores de la Escuela de Ciencias Políticas habían sido formados en otras disciplinas. Desde 1990 existe la Maestría en Ciencias Políticas en la Universidad de Costa Rica y se han desarrollado esfuerzos importantes por impulsar a jóvenes profesores para que continúen estudios en universidades europeas, latinoamericanas y norteamericanas. Asimismo, en 1998 se creó el Doctorado en Gobierno y Políticas Públicas, con el apoyo conjunto de las Escuelas de Administración Pública y la Escuela de Ciencias Políticas de la Universidad de Costa Rica; así como de la Maestría Centroamericana en Administración Pública y la Maestría en Ciencias Políticas. Por otra parte, no existen en el país publicaciones periódicas especializadas en ciencia política, por lo general, los investigadores hacen uso de diferentes revistas científicas nacionales (Revista de Ciencias Sociales, Anuario de Estudios Centroamericanos y Revista Parlamentaria, entre otras) para dar a conocer los resultados de investigación.

\section{REFLEXIONES FINALES}

Este artículo ha efectuado una primera compilación de estudios en ciencia política producidos mayoritariamente en el país desde la fundación de la Escuela de Ciencias Políticas de la Universidad de Costa Rica en 1968. Pese a no ser una compilación exhaustiva, y a la generalidad en los criterios de análisis empleados, ha sido posible identificar algunas tendencias en los temas y estilos de investigación política.

Las conclusiones pueden resumirse como sigue: la mayoría de la producción intelectual data de quince años 0 menos $^{11}$. En la práctica, la ciencia política es una disciplina muy joven en el país. Hay una concentración temática en la materia electoral y de partidos políticos, a pesar de que se ha venido

10 El estudio constituye la sexta entrega de resultados de seguimiento de graduados que periódicamente realiza la Oficina de Planificación de la Educación Superior perteneciente al Consejo Nacional de Rectores, que agrupa a las cuatro universidades estatales. El objetivo de la investigación es conocer la situación del mercado laboral de los graduados universitarios y las características del ejercicio profesional de éstos. De un total de 9013 graduados en el 2001, se seleccionó una muestra de 4.253, los cuales fueron entrevistados en el año 2002.

11 Debido a que la base de datos tiende a tener mayores omisiones a la hora de registrar las obras publicadas en la década de 1970 y 1980, es posible que las proporciones específicas reportadas en las Tablas 1 y 2 no sean precisas. 
ampliando el abanico temático. Las técnicas documentales han prevalecido, con una importante frecuencia de extracción y trascripción de datos secundarios. Existe muy baja aplicación de métodos cualitativos o cuantitativos en el estudio de la materia política. Creemos que esto nos autoriza a decir que se trata de una disciplina que muestra aún un desarrollo metodológico y técnico incipiente.

Infortunadamente, en este artículo no se exploró la vinculación de los estudios políticos en Costa Rica (aunque no exclusivamente sobre ella) con las teorías de política comparada, relaciones internacionales y la teoría política "pura". Esto es una asignatura pendiente. Al inicio de esta corta pieza se apuntó que, como tendencia, los estudios políticos se independizaron de, por una parte, la teoría sociológica de las clases sociales y, por otra, la historiografía a lo largo de la década de 1980. Sin embargo, los rumbos actuales de la reflexión política en Costa Rica no han sido sistemáticamente tratados. Un estudio de este tipo permitiría examinar su vinculación con los principales debates teóricos que movilizan las ciencias políticas contemporáneas en los principales centros de América y Europa.

Finalmente, puede argumentarse que tanto la disciplina como su desarrollo organizacional en Costa Rica se encuentran en un proceso de búsqueda de identidad, en el cual se identifican avances y limitaciones. En materia de avances se reconoce el esfuerzo sostenido de construcción de comunidad académica y disciplinaria, así como la existencia de un programa de estudios de postgrado con una trayectoria de 15 años. Por su parte las limitaciones radican en la necesidad de desarrollar estudios que profundicen en las características del mercado laboral, la existencia de revistas y publicaciones periódicas especializadas en las cuales publicar y el perfeccionamiento teórico y metodológico, principalmente en lo que se refiere a la aplicación de técnicas de investigación.

\section{REFERENCIAS}

Aguilar, Marielos. 1989a. Clase trabajadora y organización sindical. San J osé: ICES - Editorial Porvenir - FLACSO.

Aguilar, Marielos. 1989b. Los derechos civiles en Costa Rica, 1940-1980: historia de un proceso democrático. San J osé: Instituto Costarricense de Estudios Sociales.

Araya, Carlos. 1982. Liberación Nacional en la historia política de Costa Rica 1940-1980. San José: Editorial Nacional de Textos.

Arias Sánchez, Oscar. 1971. Grupos de presión en Costa Rica. San J osé: Editorial Costa Rica.

Arias Sánchez, Oscar. 1977. Caminos para el desarrollo de Costa Rica. San José: Centro de Estudios democráticos de América Latina.

Booth, John. 1995. "Elites and Democracy in Central America". En Elections and Democracy in Central America, editado por Mitchell Seligson y J ohn Booth. Chapel Hill, Estados Unidos: University of North Carolina Press, 244-263.

Booth, John. 1998. Costa Rica: Quest for Democracy. Boulder: Westview Press.

Booth, John y Mitchell Seligson. 1994. "Political Culture and Democratization: Evidence from Mexico, Nicaragua, and Costa Rica". En Political Culture and Democracy in Developing Countries, editado por Larry Diamond. Boulder: Lynne Reinner, 107-138.

Booth, J ohn y Mitchell Seligson 2005. "Political Legitimacy and Participation in Costa Rica: Evidence of Arena Shopping". Political Research Quarterly, por aparecer.

Camacho, Daniel. 1977. ¿Por qué persiste el juego democrático en Costa Rica? Heredia: Universidad Nacional.

Carvajal, Mario. 1978. Actitudes políticas del costarricense: análisis de opinión de dirigentes y partidarios. San J osé: Editorial Costa Rica.

Cerdas, Rodolfo. 1972. Crisis de la democracia liberal en Costa Rica: interpretación y perspectiva. San J osé: Editorial Universitaria de Centroamérica. 
Cerdas, Rodolfo. 1974. Por un Estado de democracia nacional. San J osé: Centro de Estudios Democráticos en América Latina.

Clark, Mary. 2001. "Costa Rica: Portrait of an Established Democracy". En Citizen Views of Democracy in Latin America, editado por Roderic Camp. Pittsburgh: University of Pittsburgh Press, 73-89.

Consejo Nacional de Rectores. 1986. Estudio exploratorio del mercado de trabajo en el campo de las ciencias políticas. San J osé: Consejo Nacional de Rectores, Oficina de Planificación de la Educación Superior.

Consejo Nacional de Rectores. 2004. La situación laboral y otras características de los graduados de las universidades estatales. San José: Consejo Nacional de Rectores, Oficina de Planificación de la Educación Superior.

Daremblum, Jaime y Samuel Stone. 1975. "Dos casos de desarrollo institucional y sus implicaciones para la práctica y la teoría social (primera parte)". Revista de Ciencias Sociales (9): 5-31.

Escuela de Ciencias Políticas. 2001. Propuesta de Plan de Estudios en Bachillerato y Licenciatura en Ciencias Políticas. Documento interno de trabajo.

Fournier, Marco. 2002. "Una tipología de los electores". Revista de Ciencias Sociales 4 (98): 9-18.

Fournier, Marco Vinicio, Ana Lucía Gutierrez y Carlos Cruz. 2002. "Resquebrajándose una tradición electoral". Revista de Ciencias Sociales 4 (98): 57-69.

Lehouq, Fabrice. 1992. "Conflicto de clases, crisis política y destrucción de las prácticas democráticas en Costa Rica. Reevaluando los orígenes de la Guerra Civil de 1948". Revista de Historia 25 (Enero - junio 1992): 65-96.

Lehouq, Fabrice. 1995. "La dinámica política institucional y la construcción de un régimen democrático: Costa Rica en perspectiva latinoamericana". En Identidades nacionales y Estado moderno en Centroamérica, editado por Arturo Taracena y J ean Piel. San J osé: Editorial Universidad de Costa Rica, 281.

Lehouq, Fabrice. 1998. Instituciones Democráticas y Conflictos Políticos en Costa Rica. Heredia: Editorial Universidad Nacional Autónoma.

Mahoney, James. 2001. The Legacies of Liberalism. Baltimore: J ohns Hopkins University.

Molina, Ivan y Fabrice Lehouq. 1999. Urnas de lo inesperado. San J osé: Editorial Universidad de Costa Rica.

Peeler, J ohn. 1985. Latin American Democracies: Colombia, Costa RIca, Venezuela. Chapel Hill, Estados Unidos: University of North Carolina Press.

Peeler, John. 1991. "Elite Settlements and Democratic Consolidation". En Elites and Democratic Consolidation in Latin America and Southeastern Europe, editado por J ohn Highley y Richard Gunther. New York: Cambridge University Press, 81-112.

Programa Estado de la Nación. 2001a. Auditoría ciudadana sobre la calidad de la democracia. San José: Editorama.

Programa Estado de la Nación. 2003b. Segundo Informe sobre desarrollo humano en Centroamérica y Panamá. San José: Editorama.

Programa Estado de la Nación. 2004. X Informe Estado de la Nación en Desarrollo Humano Sostenible (2003). San José: Editorama.

Raventós Vorst, Ciska, Marco Vinicio Fournier Facio, Olman, Ramírez Gutiérrez, et al. 2004. El Abstencionismo en Costa Rica. ¿Quiénes son y por qué no votan los ciudadanos en las elecciones nacionales? San J osé: Instituto de Investigaciones Sociales de la Universidad de Costa Rica.

Rodríguez, Florisabel y Sylvia Castro. 2002. "Fracturas del apoyo al sistema político costarricense". Revista de Ciencias Sociales 4 (98): 19-28.

Rodríguez, Florisabel, Sylvia Castro y Rowland Espinoza. 1998. "La intolerancia anda suelta. El estado actual de la cultura política costarricense". En El sentir democrático: estudios sobre cultura política centroamericana, editado por Florisabel Rodríguez, Sylvia Castro y Rowland Espinoza. Heredia: Editorial Universidad Nacional Autónoma, 283-335.

Rodríguez, Florisabel, Sylvia Castro y J ohnny Madrigal. 2004. "Desde la mirada de la juventud. valoración de la longeva democracia costarricense". En Herencia de paz, editado por Florisabel Rodríguez, Sylvia Castro y Johnny Madrigal. Heredia: Editorial Fundación Universidad Nacional Autónoma, 455-531.

Rojas, Manuel. 1990. "Los partidos políticos y las perspectivas de la democracia en Costa Rica”. Abra 4: 10-30.

Rojas, Manuel (ed.). 1989. Costa Rica: la democracia inconclusa. San José: Departamento Ecuménico de Investigaciones.

Rovira, Jorge. 1988. Estado y política económica en Costa Rica: 1948-1970. San José: Editorial de la Universidad de Costa Rica. 
Salazar, Jorge. 1981. Política y reforma en Costa Rica, 1914-1958. San J osé: Editorial Porvenir.

Salazar, Jorge. 1990. "El modelo político electoral de la democracia costarricense, 1920-1980". Revista de Ciencias Sociales (48): 17-30.

Seligson, Mitchell. 1980. "Trust, Efficacy, and Modes of Political Participation: A Study of Costa Rican Peasants". British Journal of Political Science 10 (1): 75-98.

Seligson, Mitchell. 1999. "Costa Rican Exceptionalism: Why Ticos Are Different". En Democracy Through Latin American Lenses: Citizen Views from Mexico, Chile and Costa Rica, editado por Camp, Roderic. New Orleans: Tulane University, 90-106.

Seligson, Mitchell. 2002. "Trouble in Paradise: The Impact of the Erosion of System Support in Costa Rica, 1978-1999". Latin America Research Review 37 (1): 160-185.

Seligson, Mitchell y J ohn Booth. 1993. "Political Culture and Regime Type: Evidence fron Nicaragua and Costa Rica". J ournal of Politics 55 (3): 777-792.

Seligson, Mitchell y Miguel Gómez. 1987. "Elecciones ordinarias en tiempos extraordinarios: la economía política del voto en Costa Rica". Anuario de estudios centroamericanos 13 (1): 71-92.

Seligson, Mitchell y Edward Muller. 1990. "Estabilidad democrática y crisis económica: Costa Rica 1978-1983". Anuario de estudios centroamericanos 16-17 (2-1): 71-92.

Sojo, Ana. 1984. Estado empresario y lucha política. San José: EDUCA.

Urcuyo, Constantino. 2003. Reforma política y gobernabilidad. San J osé: Editorial J uricentro.

Vega Carballo, J osé Luis. 1978. La crisis de los partidos políticos tradicionales de Costa Rica. San J osé: Academia Costarricense de Bibliografía.

Vega Carballo, J osé Luis. 1982. Poder político y democracia en Costa Rica. San J osé: Editorial Porvenir.

Vega Carballo, J osé Luis. 1986. Hacia una interpretación del desarrollo costarricense: ensayo sociológico. San J osé: Editorial Porvenir.

Wilson, Bruce. 1994. "When Social Democrats Choose Neoliberal Economic Policies: The Case of Costa Rica". Comparative Politics 26 (2): 145-168.

Wilson, Bruce. 1998. Costa Rica: Politics, Economics, and Democracy. Boulder: Lynne Rienner Publications.

Wilson, Bruce. 2004. "The Best Laid Schemes... Gang Aft A-Gley: J udicial Reform in Latin America-Evidence from Costa Rica". Journal of Latin American Studies 36 (3): 507-531.

Wilson, Bruce y Juan Carlos Rodríguez Cordero. 2004. Legal Opportunity Structures and Social Movements: The effects of Institutional Change on Costa Rican Politics (todavía sin publicar). Orlando.

Yashar, Deborah. 1997. Demanding Democracy. Stanford: Stanford University Press.

Ronald Alfaro Redondo es Licenciado en Ciencias Políticas, Universidad de Costa Rica (UCR). Es profesor de la Escuela de Ciencias Políticas (UCR) e investigador del Programa Estado de la Nación auspiciado por el Consejo Nacional de Rectores (CONARE) y la Defensoría de los Habitantes de la República. Especializado en temas sobre gobierno, comportamiento electoral y democracia local.

(E-mail: ralfaro@estadonacion.or.cr)

J orge Vargas Cullell es candidato a Ph.D. Departamento de Ciencia Política, Universidad de Notre Dame (EE.UU) y Licenciado en Sociología por la Universidad de Costa Rica. Actualmente es Director Adjunto del Programa Estado de la Nación. Además, trabaja el tema de calidad de la democracia, tiene experiencia como consultor para entidades internacionales y ha laborado en la administración pública.

(E-mail: jorgevargas@estadonacion.or.cr) 